\title{
3.8 HOSPITALIST AS EDUCATOR
}

Hospitalists serve as educators and role models for all members of the multidisciplinary care team, including student learners, fellow physicians, allied health professionals, and hospital administrators. "Hospitalist as educator" refers to specific interactions with these team members to educate them about a wide range of knowledge and clinical skills such as patient care plans, treatment protocols, aspects of patient safety, and evidence-based problem-solving exercises. In this role as educators, hospitalists facilitate team building. They instruct students in an optimal learning environment, provide feedback, and promote independent thinking. They model efficient clinical decision-making and communication skills during physician-patient encounters. Hospitalists must attend to the learning needs of a generation of medical trainees that has an affinity for technology, interaction, and group-based learning, while also operating in an environment of restricted resident work hours. The hospitalist as educator core competency is essential to effecting organizational excellence.

\section{KNOWLEDGE}

Hospitalists should be able to:

- Explain the role of the hospitalist as an educator.

- Describe adult education principles.

- Explain factors that may facilitate or inhibit learning.

- Define the concept of a teachable moment.

- Describe the benefits and limitations of various teaching modalities.

- Identify resources for training materials.

- Describe the process of developing a formal educational session, including performing a needs assessment, determining teaching goals and objectives, developing teaching materials and activities, and evaluating a learner's comprehension of the target material.

- Describe practical steps for delivering dynamic presentations for multiple venues, including bedside teaching to trainees, small group discussions with coworkers or managers, academic detailing for new initiatives, and didactic lectures at national meetings.

- Describe models for clinical teaching (eg, the "microskills" model).

- Explain the process of applying competencies to curricular development.

\section{SKILLS}

Hospitalists should be able to:

- Establish a comfortable and safe learning environment.

- Establish expectations for each teaching session and clearly articulate the objectives.

- Determine the information needs of the intended recipient and tailor messages to the needs, abilities, and preferences of the intended recipient.

- Effectively assess learners' progress towards the goals of the teaching session.
- Frame educational interventions in a manner that sets up trainees for success.

- Provide prompt, explicit, and action-oriented feedback in a manner conducive to self-improvement.

- Facilitate learners' self-assessment of comprehension of target information and development of plans for further self-education.

- Promote evaluation standards that are fair and facilitate personal and professional development.

- Instruct at the level of learners' experience and knowledge and accommodate for learners at different levels.

- Seek feedback on the effectiveness of instruction methods, modalities, and materials.

- Encourage and provide tools for lifelong, self-directed learning and clinical problem-solving.

- Structure the timing and delivery of information and learning experiences to maximize comprehension.

- Use adult learning principles in the development or selection of educational programs, methods, and materials.

- Promote the effective use of the "teachable moment" in the education of patients, students, and healthcare professionals.

- Use explicit and accessible language to explain clinical decision-making to learners.

- Make the clinical reasoning process understandable, explicit, and relevant to learners.

- Promote efficient, up-to-date clinical problem-solving during every patient encounter.

- Model the integration of quality initiatives and patient feedback into clinical decision-making.

- Provide bedside teaching that is informative and comfortable for patients, trainees, and members of the multidisciplinary care team.

- Demonstrate effective mentoring, including role modeling and active feedback techniques.

- Demonstrate procedures by explaining indications and contraindications, equipment, each sequential step in the performance of the procedure, and necessary follow-up.

- Lead, coordinate, and/or participate in efforts to formulate a needs assessment program for hospitalists' continued professional development.

- Lead, coordinate, and/or participate in educational scholarship.

\section{ATTITUDES}

Hospitalists should be able to:

- Project enthusiasm for the teaching role.

- Respect learners from all backgrounds, knowledge, and skill levels.

- Promote an atmosphere of cooperation among patients, trainees, and multidisciplinary team members.

- Advocate the importance of lifelong learning and mentorship.

- Advocate the dual role of all healthcare professionals as 
simultaneous educators and students.

- Balance patient care and teaching regarding relevant time constraints.

- Promote an organizational environment in which knowledge deficiencies are identified and targeted.

- Establish a trusting relationship with patients and families, medical trainees, and the multidisciplinary team.

- Admit the limitations of one's knowledge and respond ap- propriately to mistakes.

- Reflect on teaching moments to identify opportunities for improvement.

- Promote evidence-based information acquisition and clinical decision-making.

- Use the role of the "hospitalist as educator" to lead, coordinate, and/or participate in performance improvement initiatives. 